\title{
DEVELOPING A CLIMATOLOGY OF CIRRUS LIDAR RATIOS USING UNIVERISTY OF WISCONSIN HSRL OBSERVATIONS
}

\author{
Ralph Kuehn ${ }^{1}$, Robert Holz ${ }^{1}$, Edwin Eloranta ${ }^{1}$, Mark Vaughan ${ }^{2}$, and Johnathan Hair ${ }^{2}$ \\ ${ }^{1}$ Cooperative Institute for Meteorological Satellite Studies (CIMSS), University of Wisconsin-Madison, \\ WI 53706, USA,
}

${ }^{2}$ NASA Langely Research Center, Hampton, VA 23681-2199

*Email: ralph.kuehn@ssec.wisc.edu

\begin{abstract}
Measurements of ice cloud lidar ratio from the University of Wisconsin High Spectral Resolution Lidar System (UW HSRL) are shown, for the period 2013/06/18-2013/11/04 in Hunstville, AL. These data were acquired as part of the SEAC4RS campaign. The layer-averaged median lidar ratio , at $532 \mathrm{~nm}$, for non-precipitating ice-clouds, $0.3<$ OD $<3.0$, observed during the experiment was determined to be $25.7+/-10.6$ sr. As part of this work we've also developed an automated cloud and precipitation classification and detection algorithm.
\end{abstract}

\section{INTRODUCTION}

Our ability to detect and probe the vertical extent of cirrus was hugely improved with the launch of the NASA- CNES (CALIPSO) mission in April 2006. However, our skill at retrieving the optical properties of the cirrus detected by CALIPSO is not yet commensurate with our detection abilities. As with any new observing system, CALIPSO faces challenges and uncertainties in the retrieval of the geophysical parameters from its fundamental measurements. The data acquired during NASA's many intensive airborne and ground-based field campaigns can provide new insights into these knowledge gaps that can be used to improve retrieval accuracies and, equally important, help constrain overall retrieval uncertainties using more detailed and higher signal-to- noise measurements. To improve CALIPSO optical properties retrievals, we show High Spectral Resolution Lidar (HSRL) measurements acquired during the SEAC4RS campaign from Huntsville, AL, USA. Because the extinction and optical depth retrievals for elastic backscatter lidars like CALIOP typically rely on a priori assumptions about layer-mean extinction- to- backscatter ratios (AKA lidar ratios), uncertainties are high. In contrast, HSRLs can directly determine ice cloud extinction and lidar ratio by separately measuring the molecular and particulate components of the total backscattered signal, thus largely eliminating many of the uncertainties inherent in elastic backscatter retrievals. During the SEAC4RS campaign, three HSRL systems were deployed: an aircraft system on the DC8 and two ground-based systems, this paper focuses on measurements of lidar ratios acquired during the period 2013/6/18-2013/11/4 in Hunstville, AL.

\section{METHODOLOGY}

The HSRL technique (Shipley et al., 1983, Piironen and Eloranta 1994) relies on spectral discrimination between particle and molecular backscatter in the receiver using optical filters which enables independent retrievals of backscatter and extinction without reliance on uncertain assumptions about the particle microphysics. By design the HSRL technique provides measurements that are self-calibrated requiring only the current atmospheric density profile. The University of Wisconsin's (UW) HSRL instruments are specifically designed and optimized for cirrus cloud measurements in that they have a very narrow receiver field of view (FOV) ( 100urad) which minimizes the effects of multiple scattering. The UW-HSRL system deployed in Huntsville, measured the particulate backscatter, extinction, and linear depolarization at a single wavelength $(532 \mathrm{~nm})$. The nominal resolution for the backscatter and depolarization (extinction) is 15 meters (75 meters) in the vertical, for 120 seconds of integration. The vertical coverage is from $\sim 300 \mathrm{~m}$ AGL to $30 \mathrm{~km}$. 

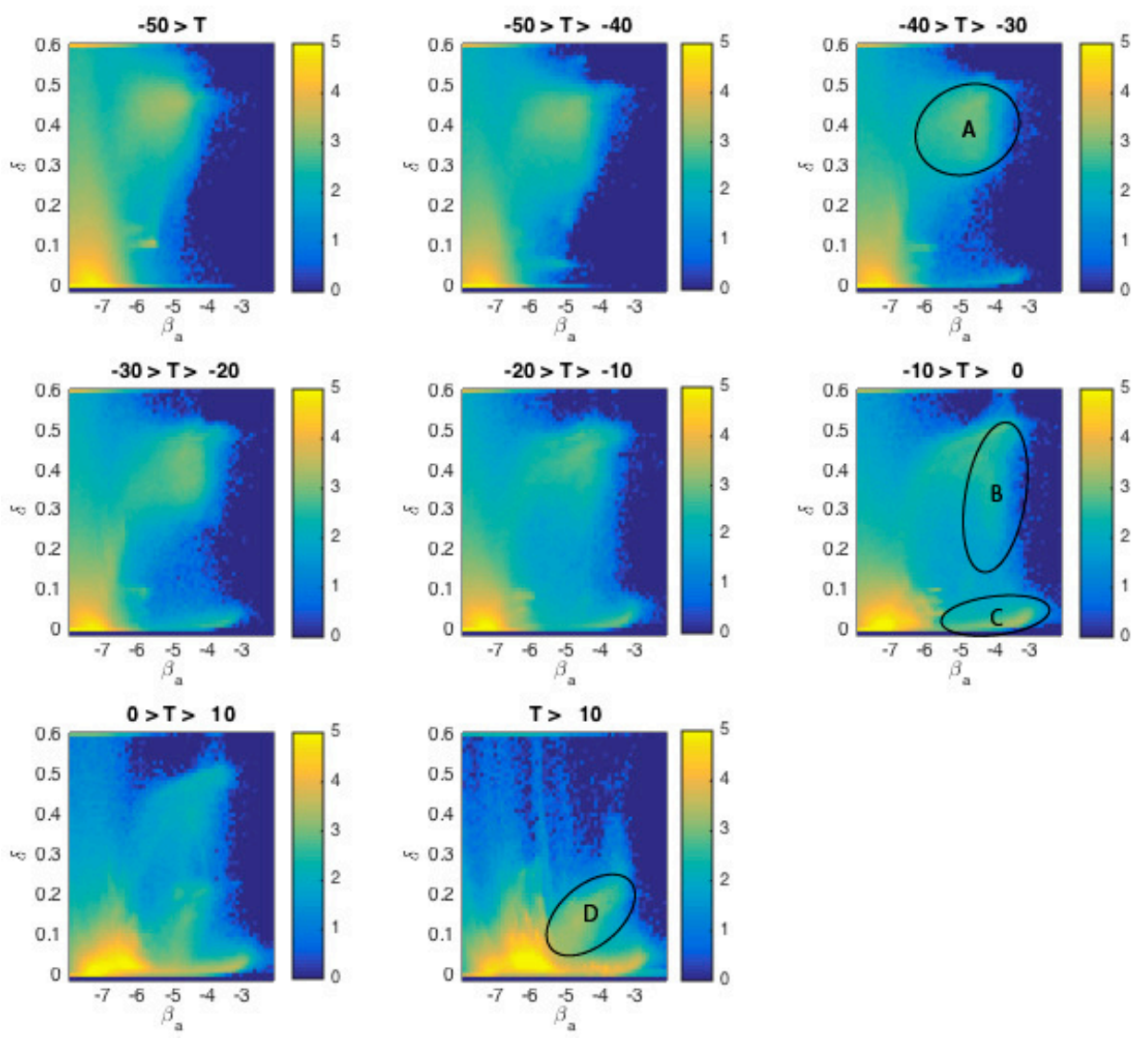

Figure 1 Two dimensional histogram of depolarization vs backscatter cross-section (log10) for different temperature regimes ( $T$ is in Celsius). Number concentration, i.e. intensity, is on a log10 scale. Annotated regions correspond to ice-clouds (A), melting ice-cloud that is precipitating (B), water clouds (C) and liquid precipitation (D). See text for further information.

For the SEAC4RS campaign a new set of QA flags, based upon expert inspection of the data, were incorporated into the data processing stream to provide data users additional information on data quality. In conjunction with the existing QC flags, which are based upon molecular-channel SNR and other system health and status information, processing and analysis of these large data sets is now tractable. Using this new quality controlled dataset we have implemented routines to classify aerosols, clouds, and precipitation in HSRL measurements.

The classification of 'features' in HSRL data is done on a range bin-by-range bin basis rather than by scanning each profile. We've found that with the typically high SNR and up-looking orientation of the HSRL this allows for more flexibility in both finding and classifying features. Depolarization ratio vs backscatter cross-section for a range of atmospheric temperature regimes, that are used to classify features as shown in Figure 1. Annotated ellipses are drawn to help the discussion and but do not necessarily indicate thresholds of the classification algorithm. Data were averaged to one minute intervals with a $30 \mathrm{~m}$ vertical range bin from 400 to $15 \mathrm{~km}$ range, QA and QC filters, in particular the molecular signal lost filter was applied. The molecular signal lost is valuable for filtering out low SNR data from above fully attenuating features.

In figure 1, non-precipitating ice clouds dominate the region indicated by ' $A$ ', these clouds are not found below the freezing level and depolarization and backscatter cross-sections are tightly constrained around 0.4 and $1 \times 10^{\wedge}(-5)\left(\mathrm{m}^{*} \mathrm{sr}-1\right)$, respectively. Depolarizing aerosols at higher altitude typically have backscatter cross-sections below $1 \times 10^{\wedge}(-6)\left(\mathrm{m}^{*} \mathrm{sr}-1\right)$. Melting ice clouds are found in the temperature range -20 to $0 \mathrm{C}$. Backscatter cross-sections are high $\sim 1.0 \times 10^{\wedge}(-4)$ 
$(\mathrm{m} * \mathrm{sr}-1)$ and depolarization are quite variable, from 0.1 to 0.55 . The variability in depolarization can be explained by the contribution from multiple scattering at high depolarizations, to large quantities of liquid drops or melting ice at lower depolarizations. Almost all of these clouds are associated with moderate to high precipitation rates. Water clouds dominate the area indicated by ' $\mathrm{C}$ ', low to no depolarization and large backscatter cross-section values. Note that the depolarization increases at very high backscatter values from the increase in multiple scattering at high extinction values. Also note that none of these water clouds are found below $-40 \mathrm{C}$. Finally, area ' $\mathrm{D}$ ' denotes liquid precipitation, these are all 'warm' features, above $0 \mathrm{C}$, and depolarization increases exponentially with backscatter because of the increase in multiple scattering from large spherical drops.

These regions are used to separate the point measurements into 4 different feature masks for the different types: water clouds, ice clouds, melting ice clouds, and precipitation. From the feature mask, top and bases of the features are determined and the layer properties of the features are calculated. Threshold values are shown below:

Water cloud: Backscatter cross-section > $1.45 \times 10^{\wedge}(-5)$, depolarization < line defined by $\mathrm{y}=\mathrm{m} * \mathrm{x}+\mathrm{b}$, where $\mathrm{m}=0.0545, \mathrm{~b}=0.2936, \mathrm{~b}$ is the $\log 10$ of backscatter cross section.

Ice cloud: Backscatter cross-section $>1.87 \times 10^{\wedge}(-$ 5), $0.3<$ depolarization $<0.58, \mathrm{~T}<-5 \mathrm{C}$.

Ice cloud, precipitating: Backscatter cross-section $>1 \times 10^{\wedge}(-5), 0.26<$ depolarization $<0.53, \mathrm{~T}>0 \mathrm{C}$

Precipitation: Backscatter cross-section $>1 \times 10^{\wedge}(-$ 5), $0.08<$ depolarization $<0.3, \mathrm{~T}>0 \mathrm{C}$

\section{RESULTS}

Using the classification scheme above, we've calculated the lidar ratio for ice clouds (no predicating) with optical depths greater than 0.3 and with a base height above $4 \mathrm{~km}$. These results are shown in Figure 2. The mean and median lidar ratios are 26.7 , and $25.7+/-10.6 \mathrm{sr}$ respectively.

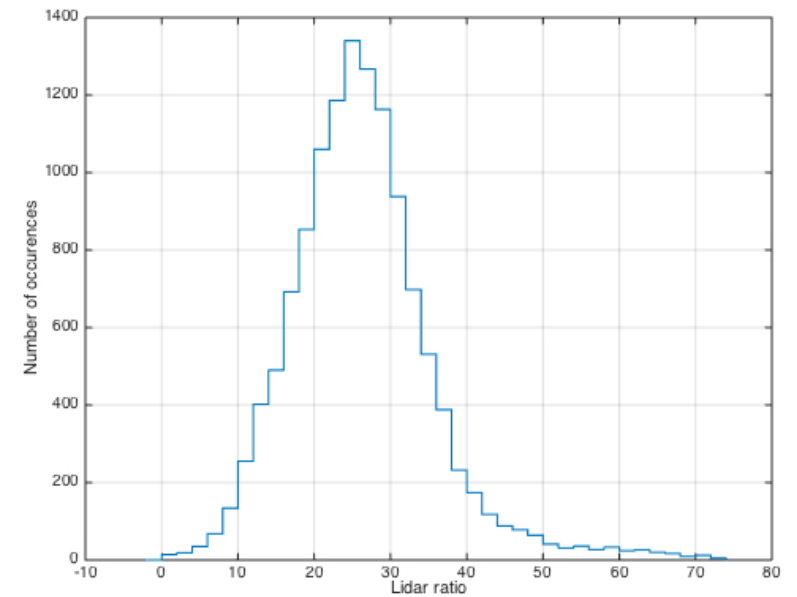

Figure 2 Measurements of lidar ratio in cirrus clouds from the UW-HSRL in Huntsville, AL, 2013/06/18-2013/11/04.

\section{SUMMARY}

In this paper we have shown first results of ice cloud lidar ratios measured with the UW HSRL lidar system in Huntsville, AL during SEAC4RS. These results are the first part of the analyses of the data set. With the addition of improvements to the feature classification scheme, adding corrections for multiple scattering, and a detailed error analyses we can then look at profiles of lidar ratio measurements, and investigate the relationships between the lidar ratio and other optical parameters measured by the HSRL.

\section{ACKNOWLEDGEMENT}

This work is supported by NASA grant NNX15AD08A.

\section{REFERENCES}

[1] Shipley, S. T., D. H. Tracy, E. W. Eloranta, J. T. Trauger, J. T. Sroga, F. L. Roesler and J. A. Weinman, 1983: A High Spectral Resolution Lidar to Measure Optical Scattering Properties of Atmospheric Aerosols, Part I: Instrumentation and Theory, Appl. Optics, 23, 3716-3724, doi:10.1364/AO.22.003716.

[2] Piironen, P., and E.W. Eloranta 1994: Demonstration of a high-spectral-resolution lidar based on an iodine absorption filter, Optics Letters, 19, 234-236. 